\title{
Contact tracing e contact tracer
}

\author{
Miriam Di Carlo
}

PUBBLICATO: 11 GENNAIO 2021

L a pandemia che ha coinvolto l'intera umanità nel 2020 ha contribuito senz'altro all'introduzione e diffusione di molte parole nuove nelle varie lingue e, quindi, anche in italiano: basti pensare a lockdown, droplet, termoscanner, distanziamento sociale, didattica a distanza, persino coronavirus; sono tutte parole che, prima sconosciute alla maggior parte dei parlanti, sono in breve tempo entrate nel lessico quotidiano. A questa lista va aggiunta la locuzione contact tracing con cui si indica 'il processo di identificazione e monitoraggio delle persone entrate in contatto con un individuo affetto da una malattia infettiva (persona indice)'. Di solito il protocollo prevede che, dopo aver individuato una persona positiva a un test diagnostico, le si chieda di indicare tutte le persone con cui è entrata in contatto nelle ore antecedenti alla manifestazione dei sintomi della malattia o, nel caso di soggetti asintomatici, nelle ore precedenti il test. A questo punto si procede di solito chiamando telefonicamente (o con altro mezzo di comunicazione) i contatti ritenuti "stretti", ossia quelle persone che, essendo state esposte al contagio, potrebbero a loro volta veicolare il virus e diffondere la malattia e che, quindi, devono essere sottoposte a misure cautelative, monitorate e messe al corrente sia dei pericoli della malattia sia della profilassi da adottare.

Contact tracing è un anglismo non adattato (accolto così com'è in inglese) formato da contact 'contatto' e tracing (dal verbo to trace 'cercare') letteralmente 'ricerca del contatto', non registrato finora da nessun dizionario o repertorio lessicografico della lingua italiana. Accanto a contact tracing si rilevano diversi traducenti italiani i quali risultano, però, meno diffusi: infatti se "contact tracing" registra nelle pagine in italiano di Google 447.00o risultati, "tracciamento dei contatti" ha I68.0oo occorrenze, seguito da "ricerca dei contatti" con 58.800, "rintracciamento dei contatti" con I3.Ioo, "tracciatura dei contatti" con 6.560 e infine "rintraccio dei contatti" con 4.270 (ricerche del 26/II/2020). Tralasciando tracciamento dei contatti su cui ci si soffermerà più avanti, bisogna sottolineare che i diversi sintagmi sono stati usati da varie istituzioni o altre fonti autorevoli in testi riguardanti la pandemia: ricerca dei contatti è stato spesso usato dai maggiori esponenti dell'ISS (Istituto Superiore di Sanità), tracciatura dei contatti è invece il sintagma usato frequentemente dal commissario Domenico Arcuri (assieme a tracciamento di prossimità che però conta solo 5 occorrenze in totale, sulle pagine di Google), infine rintraccio dei contatti è la variante burocratica che ricorre sul sito del Ministero della Salute. In questi casi possiamo parlare di sintagmi che cercano di tradurre e rendere trasparente il composto inglese ma che, a quanto pare, alla fine non hanno avuto la stessa fortuna dell'anglismo stesso. Discorso a parte va fatto per tracciamento dei contatti, locuzione registrata nel 2020 all'interno del repertorio online di neologismi del portale Treccani come "azione di sanità pubblica finalizzata alla prevenzione e al contenimento della diffusione di malattie infettive, consistente nell'individuazione di persone potenzialmente infette attraverso la rilevazione dei contatti avuti con persone contagiate". Per ora tracciamento dei contatti risulta abbastanza vitale, ma non quanto contact tracing: basti vedere il grafico di Google Trends che registra le ricerche (in questo caso in Italia) effettuate sul motore di ricerca: 


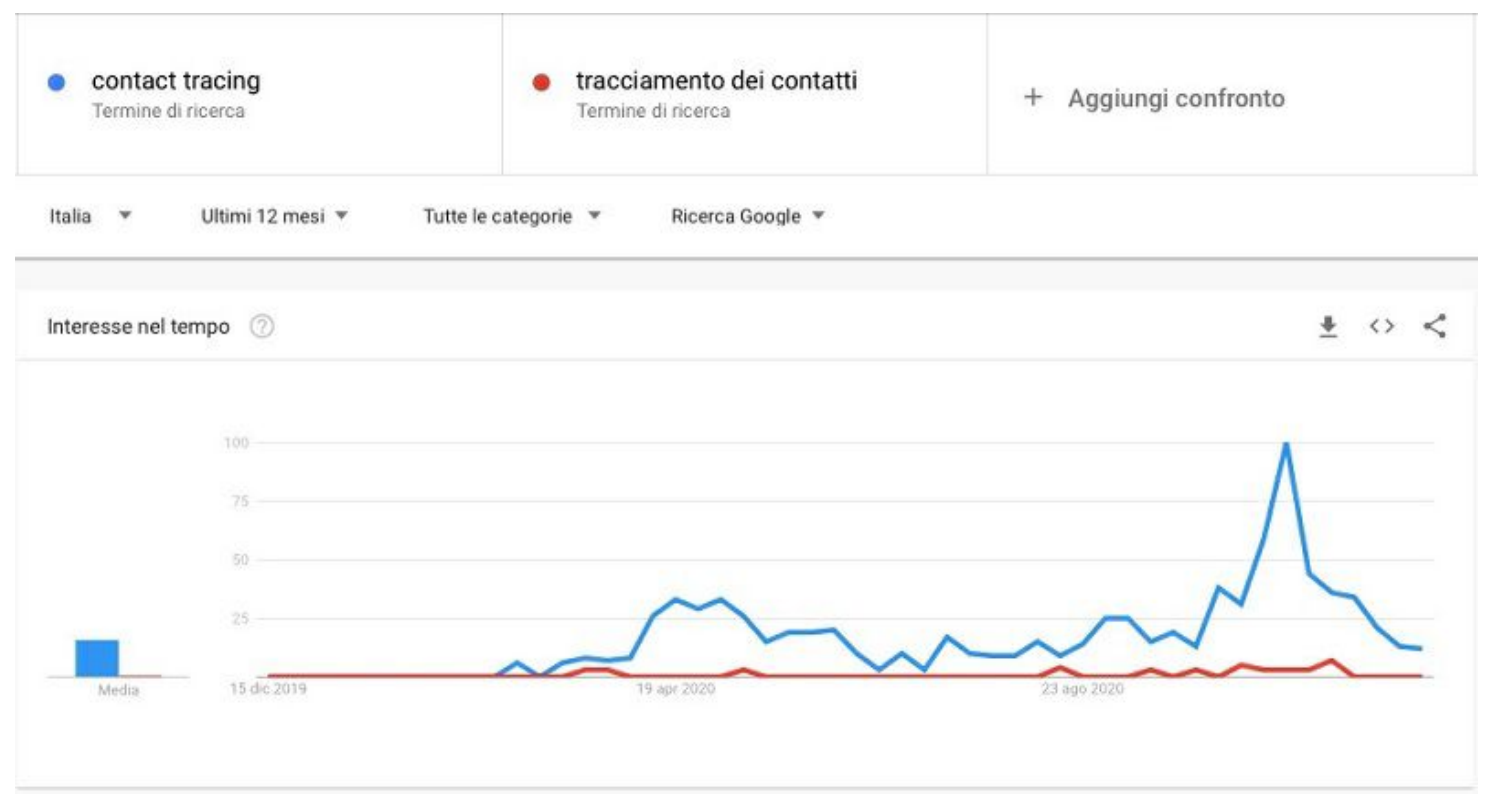

La stampa nazionale conferma questa tendenza: a titolo esemplificativo, negli articoli della "Repubblica" contact tracing ha 459 occorrenze contro le I86 di tracciamento dei contatti. Inoltre, a ulteriore conferma di questo dato vi è la diffusione di contact tracer cioè 'colui che svolge l'attività di contact tracing' che non trova concorrenti nei traducenti italiani: l'unica possibilità è tracciatore dei contatti che ha occorrenze irrisorie nelle pagine in italiano di Google contro le ir.zoo di contact tracer (ricerca del I5/12/2020).

Infine vale la pena ricordare che tracciamento ha cominciato ad assumere il significato di 'ricerca dei passaggi di un determinato prodotto e/o persona’ solo in tempi relativamente recenti: ad esempio il GDLI alla voce tracciare e tracciamento non rileva il nostro significato mentre nel supplemento del 2004 inserisce tracciabilità (a proposito dei prodotti agroalimentari) 'possibilità di conoscere tutti i passaggi attraverso i quali un cibo arriva dal produttore al consumatore' e nel supplemento del 2009 tracciabile 'che può essere ripercorso, ricostruito nelle diverse fasi del suo svolgimento'. Cosi avviene anche nel GRADIT (che registra solo tracciabilità e tracciabile con il nostro significato). È nello Zingarelli 202I e nel Devoto-Oli 202I che finalmente troviamo tracciamento rispettivamente come 'registrazione dei vari passaggi di un ciclo produttivo, di un servizio o di un'attività' e 'lindividuazione dell'origine e delle diverse fasi di un ciclo produttivo o di un servizio'.

Ma torniamo a contact tracing, che nasce nella lingua inglese all'inizio del XX secolo in relazione alla ricerca dei contatti di individui affetti da malattie infettive, soprattutto in ambito scolastico: sia l'OED che il Merriam-Webster riportano come prima attestazione il I9Io. In italiano l'anglismo comincia ad essere usato prevalentemente all'interno di testi specialistici medici tra la fine degli anni Ottanta e l'inizio degli anni Novanta in relazione alla diffusione del virus dell'HIV.

Il telefono è anche il mezzo più usato per la ricerca dei partners delle persone affette (contact tracing). (dal "Giornale Italiano di Dermatologia e Venerologia", I23, 1988, p. 56)

[...] gli oneri finanziari a carico della collettività, per un serio programma dicontact tracing, sono coerentemente tanto più elevati, quanto più è numeroso il personale da addestrare. [...] Le conclusioni sono ancor più rapide, e forse meno problematiche, per quel che concerne il contact tracing, come s'è veduto, non esistono in Italia i presupposti normativi che, ad es. in Svezia, ancorano a basi giuridicamente rigorose la possibilità di esperire, anche con mezzi compulsori, programmi o strategie di questo tipo. $\mathrm{Ma}$ - se è vero che, colà come altro, il rapporto cost-benefit e gli indici di accettabilità sociale accreditano il contact tracing di serie probabilità di successo. (da "Il Foro italiano: raccolta generale di 
giurisprudenza civile, commerciale, penale, amministrativa", I993, p. 93 е гог).

Spesso la locuzione viene associata a partner notification letteralmente 'avviso al partner' usato per indicare l'individuazione dei partner sessuali di un individuo contagiato dal virus dell'HIV e relativa notifica agli stessi. Come si può notare dai testi citati, contact tracing compare tuttavia con enfasi grafica (corsivo, parentesi, virgolette) che ne sottolinea l'estraneità rispetto alla competenza dei parlanti. Nel corso degli anni Novanta la locuzione viene associata non solo al virus dell'HIV ma a tutti i microrganismi infettivi trasmissibili (soprattutto sessualmente) come per esempio la Clamidia:

La Clamidia è l'agente più frequente causa di NGI e la sua incidenza risulta tuttora in incremento nella maggior parte dei Paesi Occidentali. Significativo e [sic] tuttavia il caso della Svezia, dove una sistematica pratica di screening, counselling e contact-tracing ha portato ad una riduzione dell'incidenza di tali forme, parallelamente all'aumento degli accertamenti diagnostici specifici. (Giampiero Carosi et alii, Malattie e trasmissione sessuale, "Caleidoscopio Italiano", I02, I996, p. 20).

Agli inizi degli anni Novanta l'anglismo comincia a essere usato anche in testi più divulgativi e di argomento non strettamente specialistico come per esempio negli articoli dei quotidiani a tiratura nazionale:

Negli Stati Uniti e in Svezia, per esempio, esistono programmi di «contact tracing», ovvero di «ricerca dei contatti» che obbligano i medici a sollecitare i pazienti sieropositivi perché rivelino i nomi dei partner a rischio e che prevedono che il paziente stesso o il medico, tramite apposite strutture, li rintraccino per invitarli agli opportuni controlli. (Cristina D’Amico, Il medico farà la spia, "Corriere della Sera", sez. Salute, $3 / 2 / 1992$, p. 20)

Sembra poi però quasi censurato nella seconda metà del decennio (forse perché tendenzialmente associato alle malattie sessualmente trasmissibili?) e negli anni Duemila se ne rintraccia una sola occorrenza significativa del zoro sul quotidiano "Corriere della Sera":

Il documento conteneva anche l'ipotesi di introdurre un sistema all'interno dei centri Aids che spingessero i pazienti risultati sieropositivi a informare i loro compagni anche attraverso la collaborazione dei medici. Meccanismo chiamato contact tracing and partner notification. (Margherita De Bac, La linea del ministero: chi è sieropositivo deve dirlo al partner, "Corriere della Sera", sez. Cronaca, 28/8/2010, p. 24)

Contact tracing ricompare con una certa frequenza tra il 2014 e il 2015, in concomitanza della diffusione nel mondo dell'epidemia causata dal virus Ebola (si veda la risposta di Maria Cristina Torchia):

Per far fronte all'epidemia di ebola in Sierra Leone, Fondazione AVSI ha lanciato una raccolta fondi con l'obiettivo di prevenire la diffusione del virus nel Paese africano, con interventi di informazione e sensibilizzazione delle comunità colpite e attività di contact tracing. (Franco Brizzo, AVSI: "Popolazione diffidente, aumenta il rischio contagio", lastampa.it, sez. Top News, 5/8/2014)

Medici Senza Frontiere è stata l'unica organizzazione al mondo in grado di gestire un centro per la gestione dei casi di ebola e in tutta questa prima fase ha operato da sola con due centri per la cura dei malati in collaborazione con le autorità locali e l'OMS per le attività di sensibilizzazione e "contact tracing", il tracciamento delle persone entrate in contatto con il malato di Ebola. (Saverio Bellizzi, Ebola, dopo la disastrosa epidemia la speranza del vaccino, lastampa.it, sez. Top News, 8/9/2015)

Il CDC sta seguendo una procedura chiamata Contact Tracing per limitare la diffusione di Ebola negli 
Stati Uniti. Questa procedura richiede agli operatori di rintracciare chiunque potrebbe essere entrato a contatto con la malattia. I possibili candidati vengono messi in quarantena in attesi che passi il periodo di sviluppo della malattia ([s.f.], I cani possono ammalarsi di Ebola?, nextquotidiano.it, I4/10/2014)

Anche in questi casi la locuzione compare frequentemente con enfasi grafica o viene spiegato all'interno del testo, spesso con qualche errore di significato:

L'Ente nazionale per l'aviazione civile (Enac) ha diffuso un comunicato in cui ribadisce agli operatori del settore (Assaeroporti, gestori aeroportuali, vettori nazionali stranieri operanti in Italia) di applicare "scrupolosamente" le procedure indicate dal ministero della Salute in merito alla prevenzione e gestione di eventuali casi sospetti di ebola. In particolare, Enac ha sottolineato l'importanza delle prassi di 'contact tracing', le schede di individuazione dei passeggeri ai fini di sanità pubblica e, soprattutto per gli aeroporti gli aspetti relativi alle informazioni da fornire ai passeggeri in arrivo e in partenza. ([s.f.], Ebola, Enac diffonde linee guida per aeroporti, lapresse.it, I6/Io/20I4)

Dopo questo significativo rilancio durato circa due anni ma di cui non si hanno tracce allinterno dei testi normativi nazionali, le occorrenze di contact tracing tornano ad essere sporadiche e occasionali: una soltanto nel 2017 sul quotidiano "la Repubblica", senza enfasi grafica e spiegazione del significato, si riferisce all'individuazione dei contatti in un focolaio di morbillo a Vietri:

A quanto si apprende da fonti del ministero della salute, si è già risaliti ad una buona parte degli ospiti degli hotel contattando direttamente le asl di residenza, ma si ha difficoltà ad individuare alcuni ospiti perché il sistema di registrazione degli hotel non conserva l'indirizzo di residenza ma solo la città. Continuano, quindi le azioni di contact tracing dei possibili ospiti venuti in contatto con i casi di morbillo. (Focolai di morbillo in due hotel a Vietri: 3 casi, oltre 700 contatti, napoli.repubblica.it, $18 / 7 / 2017$ )

Il vero e proprio rilancio avviene nel 2020 in relazione alla pandemia causata dal virus Sars-CoV-2: ad esempio, sul quotidiano "la Repubblica", delle 495 occorrenze di contact tracing, solo una è del 2017 mentre tutte le altre sono del 2020; sul quotidiano "Corriere della Sera", delle 353 occorrenze solo 2 sono antecedenti al 2020; sul quotidiano "La Stampa", dei 287 risultati per "contact tracing" solo 2 non sono del 2020 e riguardano l'ebola, per il resto si riferiscono tutti alla pandemia di Covid-rg. In particolare, nel "Corriere della Sera" contact tracing compare nella sezione dal titolo "Tracciamento", testimoniando come l'anglismo si alterni spesso al concorrente italiano:

Fase 2 - dipendono da una eventuale ripresa dei contagi, influenzata da tre fattori che l'uomo può modificare: rispettare il distanziamento sociale, indossare le mascherine e il tracciamento dei contatti dei casi con sospetto coronavirus» perché possano essere a loro volta isolati e sottoposti a tampone.

Ma è chiaro che quest'ultimo aspetto dipende anche da come la Regione si organizzerà. E, infatti, le nuove modalità per il «contact tracing» sono al centro del lavoro della task force. (Lorenza Castagneri, Mascherine, distanziamento e tracciamento dei contatti. Ecco le tre regole per riaprire, "Corriere della Sera", $\mathrm{I} 3 / 5 / 2020$, p. 6)

Il Dipartimento guidato da Angelo Borrelli nel weekend, da sabato a lunedi, ha gestito l'ingresso nel circuito anti-Covid di I.50o nuovi medici, infermieri e studenti al terzo anno di Scienze infermieristiche e di 500 addetti alle pratiche amministrative che nei prossimi tre mesi, con un contratto da collaboratori esterni, dovranno entrare nelle diciannove regioni italiane e nelle due Province autonome per aiutare a gestire il tracciamento del virus, il contact tracing diventa nuovamente il primo problema nella diffusione incontrollata dei contagi. (Corrado Zunino, Coronavirus, ecco i 1.500 tracciatori di Covid, repubblica.it, sez. Cronaca, 28/10/2020)

Durante la cosiddetta "prima ondata" che ha interessato i mesi di marzo, aprile e maggio, la locuzione viene prevalentemente accompagnata da enfasi grafica e spiegazione. Durante quella che viene definita 
la "seconda ondata" invece, contact tracing sembra ormai essersi pienamente inserito nel lessico italiano:

\begin{abstract}
"Sugli asintomatici non si molla". Lo dice il presidente dell'Istituto superiore della Sanità Brusaferro nel corso della commissione igiene al senato. Nei giorni scorsi le Regioni avevano chiesto al Governo di scrivere, nero su bianco, che quando non si riesce a fare il contact tracing si può rinunciare a fare i test ai contatti dei positivi senza sintomi. ([s.f.], Brusaferro: "Asintomatici prima frontiera della lotta al virus. Epidemia diffusa in Italia", repubblica.it, sez. Cronaca, 28/10/2020)
\end{abstract}

A rendere la locuzione familiare agli italiani hanno contribuito le notizie, le descrizioni, gli approfondimenti e le polemiche attorno alla nuova modalità digitale di contact tracing: l'applicazione per smartphone Immuni. L'applicazione è stata descritta nei testi del Ministero della Salute, fin dal suo concepimento, come una "app di contact tracing" nonostante non svolga esattamente tutti i passaggi codificati dall'Istituto Superiore di Sanità e si basi su principi di responsabilità e privacy differenti da quelli del contact tracing realizzato dagli operatori sanitari senza la mediazione di supporti digitali:

Immuni avverte gli utenti che sono stati a stretto contatto con una persona risultata poi positiva inviando loro un messaggio di allerta che include una serie di consigli su come affrontare la situazione. [...] Ecco un esempio semplificato di come funziona il sistema di contact tracing di Immuni. (FAQ Come funziona il sistema di contact tracing di Immuni?, immuni.italia.it)

L'Italia avrà un'app per tracciare i contagi da coronavirus ed evitare o almeno contrastare l'esplosione di nuovi focolai. [...] L'app sul contact tracing italiana sarà "un pilastro importante nella gestione della fase successiva dell'emergenza", la sperimentazione sarà in alcune regioni pilota, poi verrà estesa. (Bruno Ruffilli, Coronavirus, scelta l'app per il contact tracing dei contagi in Italia: ma servirà davvero?, lastampa.it, $\mathrm{I} 7 / 4 / 2020)$

Molte condizioni necessarie per il funzionamento effettivo dell'app Immuni non si sono verificate; per questo, dopo una prima ondata di entusiasmo attorno all'applicazione, contact tracing è tornato ad indicare il procedimento canonico effettuato attraverso risorse umane: i cosiddetti contact tracer. Anche questa locuzione con cui si indica 'un addetto al contact tracing', è un anglismo non adattato (registrato come voce a sé stante nell'OED e sotto la voce contact tracing nel Merriam-Webster e nel Cambridge Dictionary, con prime occorrenze attorno agli anni Quaranta) e, come abbiamo avuto modo di osservare, non trova nella lingua italiana un traducente fortunato e vitale quanto il forestierismo. In italiano contact tracer, come tutti i prestiti integrali, rimane invariato al plurale e potrebbe essere usato come femminile con l'aggiunta di determinanti o specificatori (una contact tracer, contact tracer molto preparate), ma del femminile non si sono, per ora, trovati esempi. Di tutte le occorrenze sulle pagine in italiano di Google, la maggior parte si concentra nel 2020 nonostante la locuzione sia comparsa già sporadicamente nei testi scientifici dedicati alla profilassi sanitaria delle malattie infettive. A differenza di contact tracing, che trova una diffusione in testi divulgativi gia a partire dagli anni Novanta, contact tracer comincia a entrare nella lingua di uso comune soltanto nel 2020 e in particolare attorno al mese di maggio in seguito all'ordinanza del ministro della Salute Roberto Speranza circa l'assunzione di personale sanitario dedicato specificatamente al tracciamento dei contatti (l'ordinanza però non contiene nessuno dei due termini in questione):

La speranza di stabilizzare la riapertura è nelle mani di questi contact tracer, la figura oggi più richiesta in tutto l'Occidente. Negli Usa vogliono arruolarne roo mila, in Francia 30 mila, nel Regno Unito i8 mila e l'Italia - stando all'ultimo decreto del ministro Speranza - dovrebbe schierarne circa 6 mila. (Gianluca Di Feo, I cacciatori di contatti, cosi i tracer vigileranno sulla ripartenza, repubblica.it, 3/5/2020) 
Ancora oggi però contact tracer spesso viene inserito con enfasi grafica e seguito da spiegazione:

Il loro lavoro scatta con la scoperta di un caso positivo al coronavirus: il delicato compito, quasi da detective, di ricostruire tutte le relazioni del contagiato, dall'ambiente lavorativo a quello scolastico o familiare, con lo scopo di interrompere la catena di potenziale trasmissione del Sars-Cov2. Sono i "contact tracer", operatori dei Dipartimenti di Salute pubblica delle Aziende Usl che in questi mesi non si sono mai fermati e anzi ora, con l'aumentare dei casi di virus, lavorano già ben oltre i ritmi di marzo e della primavera. (Sara Ferrari, Contact tracer 'detective' per Covid, 'Siamo ai ritmi di marzo', ansa.it, 23/10/2020)

\section{Contact tracing nei testi normativi italiani}

Uno dei motivi che ha spinto a trattare la forma contact tracing piuttosto che tracciamento dei contatti è senza dubbio l'impiego dell'anglismo all'interno dei testi normativi italiani. La prima occorrenza risale al 20 I2 in relazione alla diffusione del virus dell'HIV nei penitenziari:

Garantire Misure ed azioni concrete per la Prevenzione delle Patologie Correlate all'uso di sostanze stupefacenti in carcere, alle pratiche sessuali, ai tatuaggi, ecc. (contatto precoce e mirato con adeguate informazioni, estesa disponibilità di farmaci sostituitivi, programmi di screening calibrati per durata di detenzione, offerta attiva di contact-tracing e partner notification, campagne vaccinali, programmi mirati alle differenze di genere, ecc.) anche attraverso procedure sperimentali. (Allegato A dell'Intesa 15 marzo 2012: Conferenza unificata Stato-regioni e Stato-città ed autonomie locali sul documento recante «Infezione da HIV e detenzione [ex. Art. 8 del decreto legislativo 28 agosto 1997, n. 28I)]

Nel 2014 compare nel portale delle notizie del sito del Ministero della Salute in relazione alla diffusione del virus Ebola (pur non trattandosi di un testo normativo stricto sensu, testimonia comunque l'utilizzo della locuzione in ambito istituzionale):

Poiché la persona era asintomatica durante il viaggio, le persone che erano a bordo degli stessi aeromobili sui quali era imbarcato il predetto soggetto non corrono rischio; sono, invece, in corso di attività di contact tracing e di sorveglianza nei confronti delle persone che sono venute in contatto con la persona mentre era sintomatica, ovvero nei due giorni intercorrenti tra il momento in cui il soggetto si era presentato al pronto soccorso e quello in cui è stato messo in isolamento. ([s.f.], Audizione del Ministro Lorenzin in aula al Senato sulla diffusione del virus Ebola, salute.gov.it, I3/10/2014)

Nel 2016 viene invece impiegato nella "Relazione al Parlamento 20ı6" sulle strategie per fronteggiare l'infezione da HIV redatta dal Ministero della Salute:

Lidentificazione delle infezioni recenti è particolarmente utile per scopi epidemiologici (diffusione dell'infezione misura delloincidenza, stima dei prevalenti, identificazione dei gruppi a rischio, futuri scenari epidemiologici), per scopi clinici (impostazione della terapia), per scopi preventivi (contact tracing, partner notification, valutazione di campagne di prevenzione), e per scopi di sanità pubblica (pianificazione sanitaria, previsioni di spesa). [Ministero della Salute, Relazione al Parlamento 2016 sullo stato di attuazione delle strategie attivate per fronteggiare l'infezione da HIV (articolo 8, comma 3, legge 5 giugno 1990, n. 135)]

Sempre il Ministero della Salute ha provveduto poi a inserire la locuzione nei glossari delle proprie pubblicazioni scientifiche:

Contact tracing (CT) Indica l'insieme delle azioni che gli operatori sanitari eseguono per rintracciare e 
contattare sistematicamente ed attivamente tutti i soggetti (partner/contatti) segnalati dalla persona indice, che hanno avuto con lei relazioni a rischio (rapporti sessuali penetrativi non protetti, scambi di siringa, scambi di liquidi biologici). (Anna Maria Luzi, Barbara Suligoi, Nuove linee per lo screening e la diagnosi delle principali malattie infettive correlate all'uso di sostanze nei servizi per le dipendenze, salute.gov.it, 2017)

Tutte le occorrenze successive sono databili al 2020 e riguardano la pandemia di Covid-I9; in particolare va ricordato l'allegato ro del Decreto del Consiglio dei Ministri del 26 aprile 2020 in cui la locuzione compare senza alcuna spiegazione o enfasi grafica.

Un esempio della disinvoltura con cui contact tracing viene usato nei testi istituzionali è nel seguente decreto-legge del 28 ottobre 2020 :

Il Ministero della salute svolge attività di contact tracinge sorveglianza sanitaria nonché di informazione e accompagnamento verso i servizi di prevenzione e assistenza delle competenti aziende sanitarie locali. A tal fine, il Ministero della salute attiva un servizio nazionale di supporto telefonico e telematico alle persone risultate positive al virus SARS-Cov-2, che hanno avuto contatti stretti o casuali con soggetti risultati positivi o che hanno ricevuto una notifica di allerta attraverso l'applicazione "Immuni" di cui all'articolo 6 del decreto-legge 30 aprile 2020, n. 28, i cui dati sono resi accessibili per caricare il codice chiave in presenza di un caso di positività. (Art. 20, comma I del decreto-legge 28 ottobre $2020 \mathrm{n}$. 137 , Ulteriori misure urgenti in materia di tutela della salute, sostegno ai lavoratori e alle imprese, giustizia e sicurezza, connesse all'emergenza epidemiologica da COVID-19)

In ambito istituzionale si collocano anche le occorrenze dell'anglismo contact tracing da parte dell'Istituto Superiore di Sanità, in particolare nella Guida per la ricerca e gestione dei contatti (contact tracing) dei casi di COVID-19 in cui sono state codificate le linee guida per lo svolgimento di questa attività. Dopo aver usato il traducente italiano ricerca dei contatti, l'ISS preferisce impiegare contact tracing:

La ricerca dei contatti (o contact tracing) è uno strumento fondamentale di sanità pubblica per la prevenzione e il controllo della diffusione delle malattie trasmissibili da persona a persona, che viene utilizzato quotidianamente per il controllo delle varie malattie infettive come la tubercolosi, il morbillo, e alcune malattie trasmesse sessualmente (es. sifilide, infezioni da HIV). Lobiettivo del contact tracing è quello di identificare rapidamente le persone esposta a casi esistenti, in quanto potenziali casi secondari, e prevenire l'ulteriore trasmissione dell'infezione. [...] Nell'ambito del COVID-Ig, il contact tracing si svolge attraverso le seguenti tre fasi: - Individuazione delle persone potenzialmente esposte al virus SARS-CoV-2 in quanto contatti di una persona infetta; - Rintracciamento dei contatti, colloquio e valutazione del rischio. Viene effettuato un colloquio con le persone individuate come contatti, per informarle che potrebbero essere state esposte ad un caso di COVID-Ig. In base alla valutazione del tipo di esposizione e del livello di rischio, i contatti vengono classificati in contatti ad alto o a basso rischio e gli vengono fornite informazioni e istruzioni dettagliate sui comportamenti da tenere e misure preventive da attuare per evitare che possano eventualmente trasmettere l'infezione ad altre persone. Monitoraggio regolare dei contatti per I4 giorni dopo l'esposizione, per assicurarsi che stiano osservando la quarantena in maniera sicura, sostenibile ed efficace per impedire la trasmissione dell'infezione e per verificare l'eventuale insorgenza di sintomi, anche lievi, e identificare rapidamente i casi secondari dell'infezione, testarli, isolarli e trattarli. (Antonietta Filia, Alberto Mateo Urdiales, Maria Cristina Rota, Guida per la ricerca e gestione dei contatti (contact tracing) dei casi di COVID-19, iss.it, $25 / 6 / 2020)$

Infine una nota riguardo all'uso dell'anglismo oltre i confini italiani. Nella Svizzera italiana, tutti i testi normativi (e di conseguenza anche quelli divulgativi) pubblicati sul sito dell'Ufficio federale della sanità pubblica della Confederazione elvetica non presentano l'anglismo non adattato ma il sintagma 
italiano tracciamento dei contatti. Anche il sito dedicato all'applicazione per smartphone SwissCovid preferisce tracciamento dei contatti, così come pure avviene nei testi che spiegano ai cittadini elvetici come funziona la procedura. Per quanto riguarda le altre maggiori lingue europee, in francese si è imposto l'anglismo (contact tracer si alterna invece a traceur de contacts) mentre in spagnolo contact tracing viene spesso sostituito da rastreo de los contactos (cosi come contact tracer $\mathrm{d}$ a rastreador de contactos).

Lascia quindi un certo amaro in bocca il fatto che in Italia tracciamento dei contatti non abbia avuto forza sufficiente per diffondersi e sostituire l'anglismo.

In definitiva, contact tracing può essere annoverato tra le nuove parole uscite dall'ambito della medicina e rese di uso comune dalla pandemia. Le speranze circa la fine della pandemia fanno pensare che al tramonto di essa, tramontino anche molte delle parole ad essa associate, tra cui contact tracing e ancor piu il meno diffuso contact tracer. Soltanto il tempo e la pazienza ci daranno risposte sul destino di queste parole.

\section{Cita come:}

Miriam Di Carlo, Contact tracing e contact tracer , "Italiano digitale", 2021, XVI, 2021/1 (gennaiomarzo)

DOI: $10.35948 / 2532-9006 / 2021.5456$ 